\title{
REVISÃO: EFEITO DA ADUBAÇÃO POTÁSSICA NA CULTURA DO MILHETO
}

\author{
Dábio Silva dos Reis; Alan Soares Machado; Halef Pereira de Oliveira; Wilian Henrique Diniz Buso \\ ${ }^{1}$ Instituto Federal Goiano - Campus Ceres, GO. E-mail: halefpgtu15@hotmail.com
}

\begin{abstract}
RESUMO
Objetivou-se através deste trabalho relatar a importância da adubação potássica para as plantas, com ênfase na cultura do milheto (pennisetum glaucum) que surgiu 4 mil e 5 mil anos a.C ao sul do deserto do Saara, sendo muito utilizado na alimentação animal, com um amplo crescimento do uso na região do centro-oeste, com função de cobertura do solo para sistema plantio direto. No entanto, essa cultura é dependente da utilização do potássio na adubação com função bioquímica apresentando efeito na ativação de vários sistemas enzimáticos, que participam dos processos de fotossíntese e respiração da planta, retratando também como é a absorção desse nutriente e suas funções na planta.

Palavras chave: fertilização; fotossíntese; sistemas enzimáticos.
\end{abstract}

\section{REVIEW: EFFECT OF POTASSIUM FERTILIZATION ON MILLET CULTURE}

\begin{abstract}
The objective of this work was to report a value of potassium fertilization for the plants, with a maize crop (pennisetum glaucum) that appeared 4,000 and 5,000 years BC south of the Sahara desert, being widely used in animal feed, with one Wide use in the mid-west region, with a soil cover function for the direct flat system. However, this culture is dependent on the use of biochemistry that has an effect on the activation of various enzymatic systems, which involves the processes of photosynthesis and respiration of the plant, also depicting how it is an absorption of nutrients and their functions in the plant.

Keyboards: fertilization; photosynthesis; enzymatic systems.
\end{abstract}

\section{INTRODUÇÃO}

Para Silva (2010), existem técnicas capazes de manter o nível nutricional adequado da dieta dos animais durante os períodos de escassez de umidade no solo. Dentre essas técnicas estão a irrigação e adubação das pastagens, a conservação de alimentos volumosos na forma de feno e silagem, utilização de bancos forrageiros e os cultivos de espécies adaptadas a condições adversas.

Dentre as espécies adaptadas a condições adversas, encontra-se o milheto (pennisetum glaucum), que surgiu entre 4 mil e 5 mil anos ao sul do deserto do Saara, que por volta do ano 2.000 a.C., foi levado a Índia e gerou genótipos distintos dos originais africanos (SHARMA, 2014).

O milheto é considerado o sexto cereal mais importante no mundo situando-se depois do trigo, do arroz, do milho, da cevada e do sorgo. E empregado tradicionalmente com dois propósitos: seus grãos são usados para consumo humano, principalmente na África e na Índia e a planta inteira sendo utilizada como alimento para os bovinos, na forma de capineira ou de pastejo, (NETTO e DURÃES, 2005).

A cultura do milheto passou a se destacar no cerrado após trabalhos de seleção iniciados em 1981. Na região Centro-Oeste houve grande expansão do seu cultivo devido ao uso do grão e a 
utilidade como cobertura morta no sistema de plantio direto (IBGE, 2014). Alvarenga et al. (2001) destacam como vantagens do milheto, o sistema radicular altamente desenvolvido, podendo alcançar mais de 2,0 $\mathrm{m}$ de profundidade, o que torna capaz de absorver nutrientes em profundidades maiores e reciclagem dos nutrientes não absorvidos pelas culturas anuais.

O milheto apresenta produção média de 7 a $10 \mathrm{t} / \mathrm{ha}^{-1}$ de matéria seca (MS) e, dependendo das cultivares, condições climáticas e fertilidade do solo, pode chegar até $20 \mathrm{t} / \mathrm{ha}^{-1}$ de MS (BOGDAN, 1977).

Mesmo sendo conhecido pela sua rusticidade, existe uma certa demanda de nutrientes básicos como o N; P e K. Segundo (Vilela et al., 2004; Curi et al., 2005), após o nitrogênio (N), o K é o nutriente requerido em maiores quantidades pelas culturas. Entretanto, diferentemente do $\mathrm{N}$ que pode ser disponibilizado por processos de fixação biológica, não existem fontes renováveis de $\mathrm{K}$, do modo que, sua disponibilidade às plantas depende essencialmente das reservas do solo e da aplicação de fertilizantes. Nas condições brasileiras, o problema agrava-se pelo fato dos solos serem pobres em minerais contendo $\mathrm{K}$ e apresentarem baixa capacidade de retenção de cátions, $\mathrm{O}$ que favorece a lixiviação do K oriundo dos fertilizantes para fora da zona de crescimento radicular.

Segundo Oliveira e Souza (2001), oficialmente as reservas de sais de potássio no Brasil são de cerca de 14,5 bilhões de toneladas na forma do minério silvinita, uma mistura de silvita (KCl) com halita $(\mathrm{NaCl})$, e do mineral carnalita $\left(\mathrm{KCl} \mathrm{MgCl}{ }_{2 \cdot 6} \mathrm{H}_{2} \mathrm{O}\right)$. Essas reservas ficam nos Estados de Sergipe e Amazonas, mais especificamente nas Bacias Sedimentares Sergipe/Alagoas e do Amazonas. Existe também uma pequena reserva no Estado do Tocantins.

Este artigo constitui-se de uma revisão bibliográfica que busca descrever a utilização e funções do potássio nas plantações dando ênfase na cultura do milheto.

\section{REVISÃO DE LITERATURA EFEITO DA ADUBAÇÃO POTÁSSICA}

A principal função do potássio na bioquímica é seu efeito na ativação de vários sistemas enzimáticos EVANS e SORGER, (1966). Carvalho et al. (1999), a ação do potássio é pouca conhecida, porém sabe-se que a folha aumenta a atividade assimiladora e a síntese de carbono, e na sua falta à fotossíntese diminui e a respiração aumenta, além de ser um elemento móvel e distribui-se por toda planta, é catalisador da passagem de aminoácidos a proteínas e diminui a susceptibilidade da planta as doenças.

Staut e Kuhiara, (1998) e Staut e Athayde, (1999), relatam que as funções fisiológicas do potássio na planta são: ação sobre o metabolismo e formação de carboidratos, quebra e translocação do amido, atuação sobre o metabolismo do nitrogênio e a síntese de proteínas, controle e regulação da atividade de vários nutrientes, neutralização de ácidos orgânicos, ativador de enzimas, promotor do crescimento de tecidos meristemáticos, e ajuste da relação entre o movimento dos estômatos e água, além de promover melhor absorção de outros nutrientes e deixar a planta menos susceptível ao ataque de pragas e doenças e mais resistente à seca. De acordo com Ernani et al. (2007) potássio tem como função a ativação de vários sistemas enzimáticos, que participam no processo de fotossíntese e respiração da planta.

A maior parte do $\mathrm{K}$ absorvido pelo milheto normalmente entra em contato com a raiz por difusão COSTA et al. (1998). Melhor resposta ao nutriente é observado em épocas com baixa disponibilidade hídrica (GRIMME, 1990). Tanto a disponibilidade hídrica (Barber, 1984) como a disponibilidade de $\mathrm{K}$ podem influenciar na morfologia radicular e no balanço entre os processos de contato do $\mathrm{K}$ e raiz (ROSOLEM et al., 2001).

As plantas dependem também do potássio para regular a abertura e fechamento dos estômatos. O próprio funcionamento dos estômatos depende de um suprimento adequado de potássio, o qual se move para dentro das 20 células-guarda. Estas acumulam água e intumescem, causando abertura dos estômatos, permitindo o movimento livre dos gases dentro e fora da 
célula. Quando o suprimento de água é baixo, o potássio é bombeado para fora das células-guarda e os poros se fecham firmemente para evitar perda de água. Se o suprimento de potássio estiver inadequado, os estômatos ficam lentos, demoram a responder e o vapor d'água é perdido (TAIZ e ZEIGER, 2009).

Segundo, Ruggiero et al. (1996), o potássio é o elemento de maior mobilidade na planta e passa com facilidade de uma célula para outra e do xilema para o floema. Por isso, é o componente mineral de maior expressão nos processos osmóticos das plantas que envolvem absorção e armazenamento de água. Para Cakmak (2005), o potássio tem atuação em vários aspectos estruturais, fisiológicos e bioquímicos da vida das plantas. Além da produtividade, a suplementação adequada contribui no reforço estrutural, tornando mais difícil a penetração de patógenos e deixando a planta menos suscetível ao acamamento.

\section{MANEJO NA ADUBAÇÃO POTÁSSICA}

Conforme, Yamada e Roberts (2005), o correto manejo da adubação potássica em relação a doses, modos, épocas e fontes a serem utilizadas, devem ser consideradas quanto aos aspectos com a exigência da cultura, o preço do fertilizante, o efeito salino sobre as plantas, o potencial de perdas (principalmente por lixiviação) que os solos tropicais apresentam e a condição físicoquímica da rizosfera influenciando a disponibilidade de potássio não trocável (NIEBES et al., 1993).

Vários estudos mostraram que a libertação de K pelas frações estrutural e não-permutável pode contribuir significativamente para o fornecimento às plantas (OBORN et al., 2005). Contudo, a taxa de libertação de $\mathrm{K}$ a partir destas formas é muito lenta e depende do processo de desagregação da rocha originária. Holmqvist et al. (2003), Kayser e Isselstein (2005) e Ogaard et al. (2002) verificaram que esta liberação é maior em solos argilosos do que em solos arenosos.

O potássio quando aplicado em grandes quantidades possui um efeito salino sobre as culturas. É recomendado para a cultura do milho e da soja a quantidade máxima de $80 \mathrm{~kg} / \mathrm{ha}^{-1}$ e o restante em cobertura, fracionando as quantidades em distintas aplicações (COMISSÃO DE QUÍMICA E FERTILIDADE DO SOLO, 2004).

Sparks e Huang (1985), destacam os fatores que interferem no movimento do potássio no solo, que são: a condutividade hidráulica, o pH do solo, o método e a taxa de aplicação do potássio durante a adubação, a umidade do solo e a absorção pela planta.

No sistema de semeadura direta, no qual o milheto vem sendo empregado, pode ocorrer maior concentração de $\mathrm{K}$ disponível nas camadas superficiais dos solos, principalmente nas linhas de semeadura da cultura anterior que, mesmo sem adubação localizada de potássio, apresenta maior concentração deste nutriente próximo às plantas do que nas entrelinhas, o que é explicado pela "lavagem" do K das plantas e descida pelo colmo, concentrando - se no solo (KLEPKER e ANGHINONI, 1995).

\section{CONSIDERAÇÕES FINAIS}

O milheto (pennisetum glaucum), apresenta grande influência na agricultura e pecuária, com exigências que devem ser supridas com as adubações potássica como em quaisquer outras culturas que tem suas exigências minerais determinada. Assim, o potássio está diretamente ligado ao desenvolvimento das plantas, com influência no sistema radicular em absorver água e evitando o acamamento das plantas. As aplicações de altas doses de potássio necessitam de cuidados, pois, podem influenciar na salinização do solo.

\section{REFERÊNCIAS}

ALVARENGA, R. C.; CABEZAS, W. A. L.; CRUZ, J. C.; SANTANA, D. P. Plantas de cobertura de solo para sistema plantio direto. Informe Agropecuário, Belo Horizonte, v.22, n.208, p.25-36. 2001. 
BARBER, S.A. Soil nutrient bioavaliability: A mechanistic approach. New York, John Willey.1984.

BOGDAN, A.V. Tropical pastures and fodder plants: grasses and legumes. London: Longman Handbooks. 1977.

CAKMAK, I. Proteção de plantas contra os efeitos nocivos do estresse de fatores ambientais. In: SIMPÓSIO SOBRE POTÁSSIO NA AGRICULTURA BRASILEIRA, Piracicaba, 2005.

COMISSÃO DE QUÍMICA E FERTILIDADE DO SOLO. Manual de adubação e calagem para os Estados do Rio Grande do Sul e Santa Catarina. 10. ed. Porto Alegre, 400p. 2004.

CARVALHO, O.S.; SILVA, O.R.R.F. da.; MEDEIROS, J.C da. Adubação e calagem. In: BELTRÃO, N.E.M de. O Agronegócio do algodão no Brasil. Campina Grande: Embrapa Algodão; Brasília: Embrapa Comunicação para Transferência de Tecnologia, v.1 p.175-210. 1999.

COSTA, N.L.; SILVA FILHO, G.N.; SENA, J.O.A.; RODRIGUES, A.N.A.; ANGHINONI, I. Mecanismos de suprimento e eficiência de absorção de potássio em soja, milho, milheto, colza e lab-lab. Pesquisa Agropecuária Brasileira. v.23. n.5. p.463-468, 1988.

CURI, N.; KÄMPF, N.; MARQUES, J.J. Mineralogia e formas de potássio em solos brasileiros. In: YAMADA, T. e ROBERTS, T.L. Potássio na agricultura brasileira. Piracicaba: Instituto da Potassa \& Fosfato, p.91-122. 2005.

ERNANI, P.R.; ALMEIDA, J.A de.; SANTOS, F.C. Potássio. In: NOVAIS, R.F.; ALVAREZ, V.H.; BARROS, N.F. de.; et al., de 1o edição Fertilidade do solo. Viçosa MG, p.551-594. 2007.

EVANS, H.J.; SORGER, G.J. Role of mineral elements with emphasis on the univalent ions. Annual

$\begin{array}{lllll}\text { Review of Plant } & \text { Physiology, }\end{array}$

https://doi.org/10.1146/annurev.pp.17.060166.000403

GRIMME, H. Development of K-fertilizer recommendation. In: COLLOQUIUM OF THE INTERNATIONAL POTASH INSTITUTE, 22., Soligork, 1990. Proceedings. Soligok, IPI, p.117-131. 1990. http://www.clubeamigosdocampo.com.br/artigo/utilizacao-do-milheto-para-alimentacaoanimal-no-periodo-de-inverno-1363 acesso em: 25 fevereiro de 2017.

HOLMQVIST, J.; OGAARD, A.F.; OBORN, I.; EDWARDS, A.C.; MATTSSON, L.E.; SVERDRUP, H. Application of the profile model to estimate potassium release from mineral weathering in Northern European agricultural soils. European Journal of Agronomy, v.20, n.1-2, p.149-163. 2003. https://doi.org/10.1016/S1161-0301(03)00064-9

INSTITUTO BRASILEIRO DE GEOGRAFIA E ESTATÍ́STICA. Produção da pecuária municipal 2013. Rio de Janeiro; 41:108p. 2014.

KAYSER, M.; ISSELSTEIN, J. Potassium cycling and losses in grassland systems: a review. Grass and Forage Science, v.60, n.3, p.213-224. 2005. https://doi.org/10.1111/i.1365-2494.2005.00478.x

KLEPKER, D.; ANGHINONI, I. Características físicas e químicas do solo afetadas por métodos de preparo e modos de adubação. R. Bras. Ci. Solo, v.19 p.395-401. 1995. 
NETTO, D.A.M.; DURÃES F.O.M. Milheto tecnologias de produção e agronegócio, EMBRAPA, Informações Tecnológicas, p.17, 2005.

NIEBES, J.F.; DUFEY, J.E.; JAILLARD, B.E.; HINSINGER, P. Release of nonexchangeable potassium from different size fractions of two highly $\mathrm{K}$ - fertilized soils in the rhizosphere of rape (Brassica napus cv Drakkar). Plant and Soil, v.155-156, n.1, p. 403-406. 1993. https://doi.org/10.1007/BF00025068

OBORN, I.; ANDRIST, R.Y.; ASKEKAARD, M.; GRANT, C.A.; WATSON, C.A.E.; EDWARDS, A.C. Critical aspects of potassium management in agricultural systems. Soil Use and Management, v.21, n.1, p.102-112. 2005. https://doi.org/10.1111/j.1475-2743.2005.tb00114.x

OGAARD, A.F.; KROGSTAD, T.E LUNNAN, T. Ability of some Norwegian soils to supply grass with potassium (K) - soil analyses as predictors of K supply from soil. Soil Use and Management, v.18, n.4, p.412-420. 2002. https://doi.org/10.1111/j.1475-2743.2002.tb00260.x

OLIVEIRA, L.A.M. de.; SOUZA, A.E. Balanço Mineral Brasileiro 2001: potássio. Disponível em: http://www.dnpm.gov.br/assets/galeriadocumento/ balancomineral2001/potassio.pdf. Acesso em: 23 de fevereiro de 2016.

ROSOLEM, C.A.; ESTEVES, J.A.F.; SILVA, R.H. Significance of mass flow and diffusion in supplying K to cotton roots as affected by liming and $\mathrm{K}$ rates. In: INTERNATIONAL SYMPOSIUM ON PLANT-SOIL INTERACTIONS AT LOW pH, 5., South Africa, 2001. Programme. África do Sul, p.48. 2001.

RUGGIERO, C.; SÃO JOSÉ, A.R.; VOLPE, C.A.; OLIVEIRA, J.C.; DURINGAN, J.F.; BAUMGARTNER, J.G.; SILVA, J.R. DA; NAKAMURA, K.; FERREIRA, M.E.; KAVATI, R.; PEREIRA, V. DE P. Maracujá para exportação: aspectos técnicos da produção. Brasília, DF: EMBRAPA. SPI, 64p. Publicações Técnicas Frupex,19. 1996.

SHARMA, P.C.; SINGH, D.; SEHGAL, D.; SINGH, G.; HASH, C.T.; YADAV, R.S. Further evidence that a terminal drought tolerance QTL of pearl millet is associated with reduced salt uptake. Environ Exp Bot. v.102 n.100. p.48-57. 2014. https://doi.org/10.1016/j.envexpbot.2014.01.013

SILVA, A.G. Fontes de fósforo na produção e composição bromatológica de cultivares de milheto forrageiro. (TESE). Goiânia: universidade federal de Goiás, Escola de veterinária e zootecnia; 2010.

STAUT, L.A.; ATHAYDE, M.L.F. Efeitos do fósforo e potássio no rendimento e em outras características agronômicas do algodoeiro herbáceo. Pesquisa Agropecuária Brasileira, Brasília, v.34, n.10, p.1839-1843, 1999. http://dx.doi.org/10.1590/S0100-204X1999001000010

STAUT, L.A.; KURIHARA, C.H. Calagem, nutrição e adubação. In: Embrapa. Centro de Pesquisa Agropecuária do Oeste. Algodão: informações técnicas. Dourados: Embrapa -CPAO, p.51-70. 1998. https://doi.org/10.1590/S0100-204X1999001000010

SPARKS, D.L.; HUANG, P.M. Physical chemistry of soil potassium. In: MUNSON, R.D. (Ed.). Potassium in agriculture. Madison: American Society of Agronomy, p.201-276. 1985.

TAIZ, L; ZEIGER E. Fisiologia vegetal. 4. ed. São Paulo, Artmed, 2009. 
VILELA, L.; SOUSA, D.M.G.; SILVA, J.E. Adubação potássica. In: SOUSA, D.M.G.; LOBATO, E. Cerrado: correção do solo e adubação. Planaltina: Embrapa Cerrados, p.169-183. 2004.

YAMADA, T.; ROBERTS, T.L. Potássio na agricultura brasileira. 3. a ed. Piracicaba, Associação Brasileira para Pesquisa da Potassa e do Fosfato (POTAFOS), 841 p. 2005. 